\title{
Pediatric urolithiasis: experience at a tertiary care pediatric hospital
}

\author{
Laura Chang Kit, MD; ${ }^{*}$ Guido Filler, MD; ${ }^{+}$John Pike, MD; ${ }^{*}$ Michael P. Leonard, MD*
}

See related article on page 387

\section{Abstract}

Objective: We aimed to determine the epidemiology, risk factors, clinical characteristics, evaluation and course of patients with urolithiasis at the Children's Hospital of Eastern Ontario to improve current diagnostic and management strategies.

Methods: This was a retrospective study of children with newly identified urolithiasis between Jan. 1, 1999, and July 31, 2004. Cases were reviewed for demographics, presentation, family history, diagnostic methods and findings, metabolic and anatomic abnormalities, management, stone analysis and stone recurrence.

Results: Seventy-two patients (40 male, 32 female; mean age $11.3 \mathrm{yr}$ ) were assessed. Mean follow-up was at 1.5 years. Eighteen patients (25\%) had a family history of stones. Flank pain (63\%) was the most common presentation. Eighty-two percent of urinalyses showed microscopic hematuria. Imaging comprised abdominal plain film radiography (56\%) and (or) abdominal ultrasonography (74\%). The mean stone size was $5 \mathrm{~mm}$. Forty-one percent (28/69) of patients who underwent metabolic investigation had an abnormality. Fourteen percent of patients (10/72) had a genitourinary anatomical abnormality. Thirtyfour patients (47\%) passed their stones spontaneously, 25 patients $(35 \%)$ required surgical intervention and 13 patients $(18 \%)$ had yet to pass their stone. The mean size of spontaneously passed stones was $4 \mathrm{~mm}$. Of 42 stones analyzed, 39 (93\%) were composed of calcium oxalate or phosphate. Seventeen (24\%) patients had stone recurrence during follow-up.

Conclusion: Pediatric patients with stones present in a manner similar to adults. Abdominal plain film radiography and ultrasonography are the preferred initial radiological investigations in children as they limit radiation exposure. Metabolic abnormalities are common and may coexist with anatomic abnormalities, therefore investigations must rule these out. One-half of patients will pass their stones spontaneously. Recurrence rates are high and long-term followup is recommended.

CUAJ 2008;2(4):381-6

\section{Introduction}

Pediatric urolithiasis is an uncommon occurrence in developed countries, with a prevalence of $1 \%-5 \%,{ }^{1}$ and accounts for about 1 in 1000 pediatric hospital admissions in North America. ${ }^{2}$ However, recent studies by several major pediatric centres in the United States have observed an increased incidence. ${ }^{3}$ Though a rare pathology, there is a high degree of morbidity associated with pediatric urolithiasis. Pediatric patients tend to form stones in a recurrent pattern, with rates of recurrence of $6.5 \%-44 \% .^{4}$ This tendency as well as the destructive nature of stone formation can quickly lead to progressive decline in renal function in the fragile pediatric kidney.

The etiology of urolithiasis in children is largely unknown, ${ }^{5,6}$ though anatomic genitourinary and metabolic abnormalities and infection are usually coexistent in this population. ${ }^{7}$ To decrease morbidity and long-term effects of pediatric urolithiasis, it is essential to assess the characteristics and etiology of the patients' stones to carefully plan an appropriate management strategy.

In this study, we sought to determine the epidemiology, risk factors, clinical characteristics, evaluation and clinical course of our pediatric stone-forming population.

\section{Methods}

Medical records of children seen at the Children's Hospital of Eastern Ontario (CHEO) emergency department, outpatient clinics or inpatient wards between Jan. 1, 1999, and July 31, 2004, classified with diagnoses of "urolithiasis" or "nephrolithiasis" were examined. Only newly diagnosed cases of urolithiasis with radiological evidence of stones were included in the study. Records were then reviewed for patient demographics, clinical presentation, family history of stone disease, urinalysis findings, radiological diagnostic methods and findings, metabolic and anatomic abnormalities, management, stone analysis and stone recurrence. The Institutional Review Board at $\mathrm{CHEO}$ approved this study.

No standard protocol or algorithm was available for investigating children with suspected urolithiasis in the outpatient setting. All children included in the study had had 
some radiological investigation (kidney, ureter, and bladder radiography, ultrasonography, intravenous pyelography, CT) that identified urolithiases. All had urinalyses performed. Unfortunately, there was no gold standard imaging test that was administered to each child. Sensitivity (true pos/[true pos + false neg]) of each test (e.g., ultrasonography in stone detection) was calculated by equating the "true positives" to the number of cases detected by ultrasonography, and equating the "false negatives" to the number of cases not detected.

All stones detected and measured by their imaging modality (multiple or single, bilateral or unilateral) were included in the calculation of the overall mean stone size. However, in calculating the mean stone size of spontaneously passed stones versus stones requiring surgical intervention, only the largest stone measurement was included in cases where there was a multiple stone burden. The 1 case of a single 40-mm bladder stone was excluded from mean calculations entirely as it was the only lower tract stone in our study.

Metabolic investigations included serum electrolytes, calcium, magnesium, phosphate and uric acid, and 24-hour urine collection for calcium, oxalate, uric acid, creatinine, citrate and cystinuria screening.

\section{Results}

The study group consisted of 72 patients, 40 boys $(56 \%)$ and 32 girls (44\%), with a mean age of 11.3 (standard deviation [SD] 5.5) years at diagnosis. Boys' mean age at diagnosis was 10.3 (SD 5.4) years and girls' was 12.6 (SD 5.2) years. The mean follow-up period for both was 1.5 (SD 1.4) years. Eighteen patients $(25 \%)$ had a positive family history for stones.

The most frequent clinical presentations were flank pain $(63 \%)$, and nausea or vomiting $(49 \%)$ (Fig. 1). Microscopic hematuria was found in $82 \%$ of urinalyses, while $40 \%$ showed pyuria and $17 \%$ were negative. Ten patients (14\%) had bacteriuria, though none of these patients had typical infection stones. The most common diagnostic radiological methods were abdominal ultrasonography $(74 \%)$ and (or) abdominal plain film radiography (56\%) (Fig. 2). The sensitivity of ultrasonography was $98 \%$ versus $41 \%$ for plain film radiography. Thirty-one percent were investigated with intravenous pyelograms, which were $95 \%$ sensitive for stone detection. Five patients $(7 \%)$ were investigated with nonenhanced CT scans, with $100 \%$ sensitivity, only after ultrasonography or radiography failed to detect calculi.

The mean stone size was $5 \mathrm{~mm}$ (range 1-22 mm),

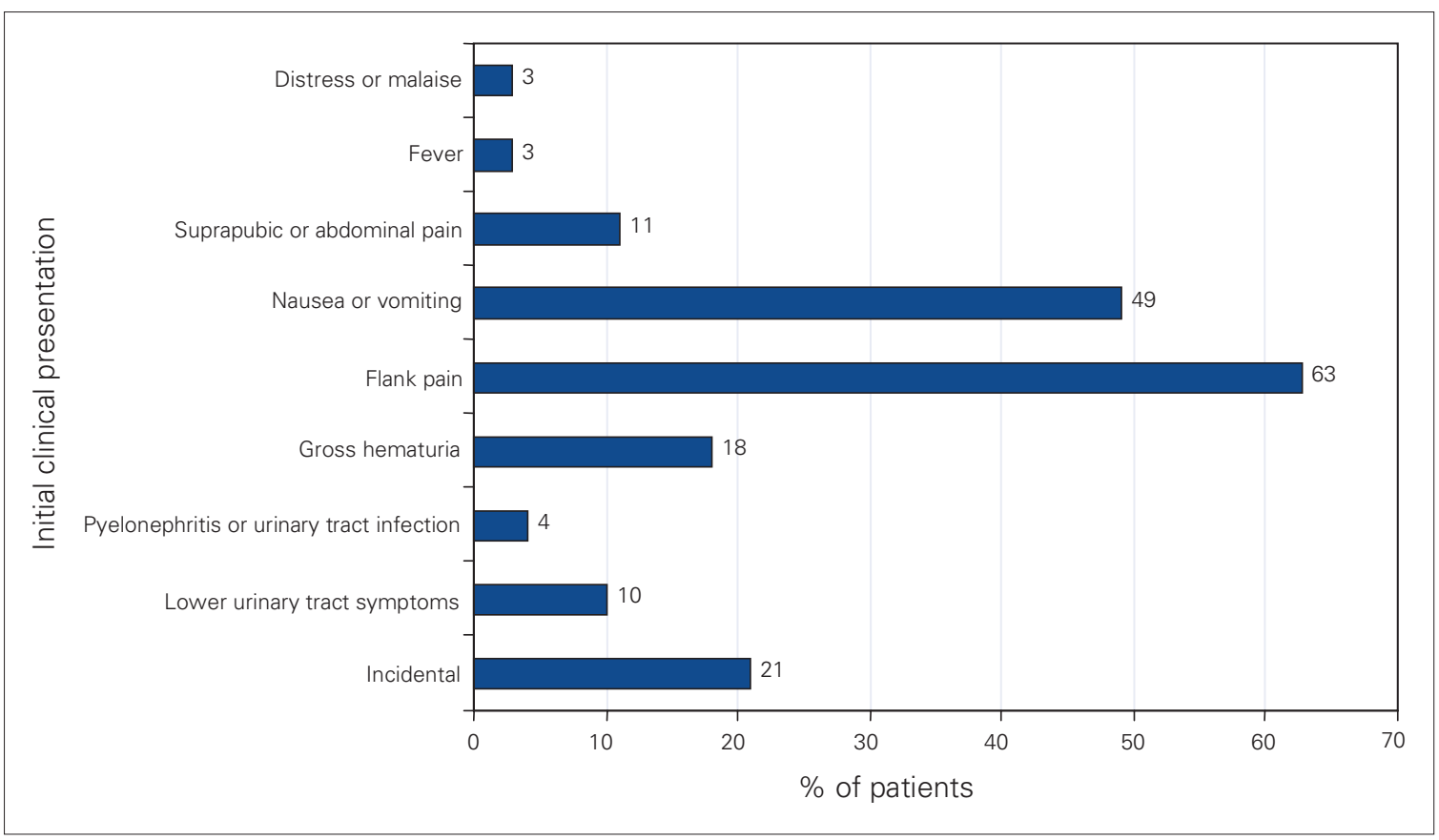

Fig. 1. Initial clinical presentations of pediatric patients with newly identified urolithiasis. 
excluding a single 40-mm bladder calculus. Fiftynine patients $(82 \%)$ presented with a single stone, while $13(18 \%)$ had multiple stones. Five patients $(7 \%)$ had bilateral disease, with 4 of 5 having associated metabolic abnormalities ( 2 hypercalciuria, 1 hypocitraturia, 1 Jacobsen syndrome). Jacobsen syndrome is a partial $11 \mathrm{q}$ deletion syndrome associated with mental and growth retardation, craniofacial, cardiac, orthopedic and genitourinary abnormalities, including cryptorchidism, collecting system duplications, horseshoe kidney and pyelectasis. Like other patients with Jacobsen syndrome, our toddler had metabolic abnormalities related to dehydration and failure to thrive, ${ }^{8}$ as well as bilateral hypodysplastic kidneys.

There was no difference in laterality in patients with unilateral stones (i.e., 34 patients right, 32 patients left). There was 1 patient who presented with a cystine bladder stone and was found to have homozygous cystinuria with no other lithiases present.

Sixty-nine patients underwent metabolic investigation, and $41 \%(28 / 69)$ of them had an abnormality (Fig. 3). Hypercalciuria was the most common metabolic abnormality, occurring in 9 patients. A total of $14 \%$ (10/72) of patients had a genitourinary anatomic abnormality (Table 1 ), with the most frequent being ureteropelvic junction obstruction ( 4 patients). These 4 patients underwent pyeloplasty; however, the other 6 patients had lesions that did not directly cause stones and thus did not undergo any specific repair to reduce stone formation.
Thirty-four patients (47\%) had spontaneous passage of their stones. The mean size of spontaneously passed stones was $4 \mathrm{~mm}$ (SD $2 \mathrm{~mm}$, range 1-11 mm), and the mean size of stones requiring surgical intervention was $9 \mathrm{~mm}$ (SD $4 \mathrm{~mm}$, range $2-22 \mathrm{~mm}$ ). This excluded the $40-\mathrm{mm}$ single bladder calculus that required cystolithotomy.

Ten of these 34 patients $(29 \%$, or $14 \%$ of total number of patients) required prescribed medical therapy. Thirteen patients $(18 \%)$ had yet to pass their stone at the time of this review. Twenty-five patients (35\%) required surgical intervention (extracorporeal shock wave lithotripsy [ESWL] 56\%, ureteroscopy $24 \%$, percutaneous nephrolithotomy $28 \%$, cystolithotomy $4 \%$ ). The only modality that failed to make patients stone-free on initial application was ESWL. Three of the 14 patients who had ESWL required a secondary surgical modality for complete stone clearance. Two of these patients underwent percutaneous nephrolithotomy, and 1 had ureteroscopic basket extraction of the residual stone.

Forty-two stones were collected and analyzed. The composition of 39 stones (93\%) was calcium oxalate or phosphate, with 2 stones $(5 \%)$ consisting of cystine and 1 ( $2 \%$ ) of uric acid. Seventeen (24\%) patients had stone recurrence during a mean follow-up of 2.8 (SD 1.3) years. The mean age at diagnosis of recurrence was 9.7 (SD 5.6) years. The majority of these patients (12 patients or $71 \%$ ) had a metabolic abnormality detected, with the most common being hyperoxaluria (Table 2).

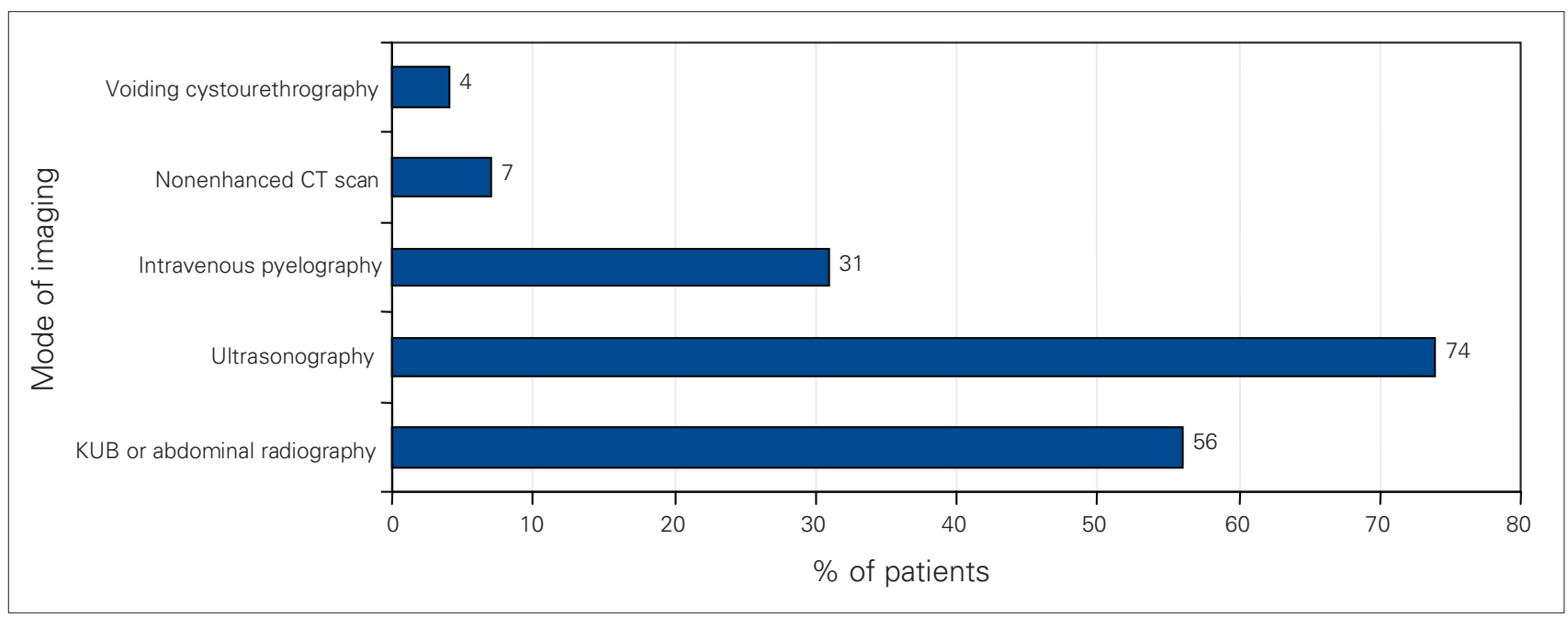

Fig. 2. Modes of imaging used to diagnose urolithiasis in pediatric patients. KUB = kidneys, ureter and bladder. 


\section{Discussion}

Pediatric stone disease varies widely in etiology, presentation, incidence and natural history depending on geographic location and economic environment. ${ }^{1,3,9-11}$ We saw an average age of presentation of about 4 years older (mean $11.3 \mathrm{yr}$ ) than reported in literature from developing countries such as Turkey, Pakistan and Armenia (mean 7.3), 1,4,6 but which correlated well with North American literature..$^{12,13}$ In keeping with other North American series, ${ }^{3,7,14}$ our series showed no significant difference between the sexes, unlike the 3:1 male-to-female ratio seen in developing countries. Series from developing countries also reported a higher incidence of stones related to infection, diet, poor nutrition and dehydration.
A child presenting with flank or abdominal pain and (or) gastrointestinal complaints (nausea, vomiting) should always be investigated with at least a urinalysis to evaluate possible stone disease. Microscopic hematuria is detected in up to $90 \%$ of children with urolithiasis, ${ }^{3}$ and we observed an $83 \%$ rate in our series. Plain film radiography (kidney, ureter, and bladder, or abdominal radiography), ultrasonography and intravenous urography have been used to diagnose urinary tract calculi, with unenhanced helical CT scan currently considered the gold-standard imaging technique. ' However, the need for sedation, concern over long-term effects of high doses of radiation exposure ${ }^{15}$ and availability of the exam are major disadvantages to CT scanning in the pediatric population. In our series,

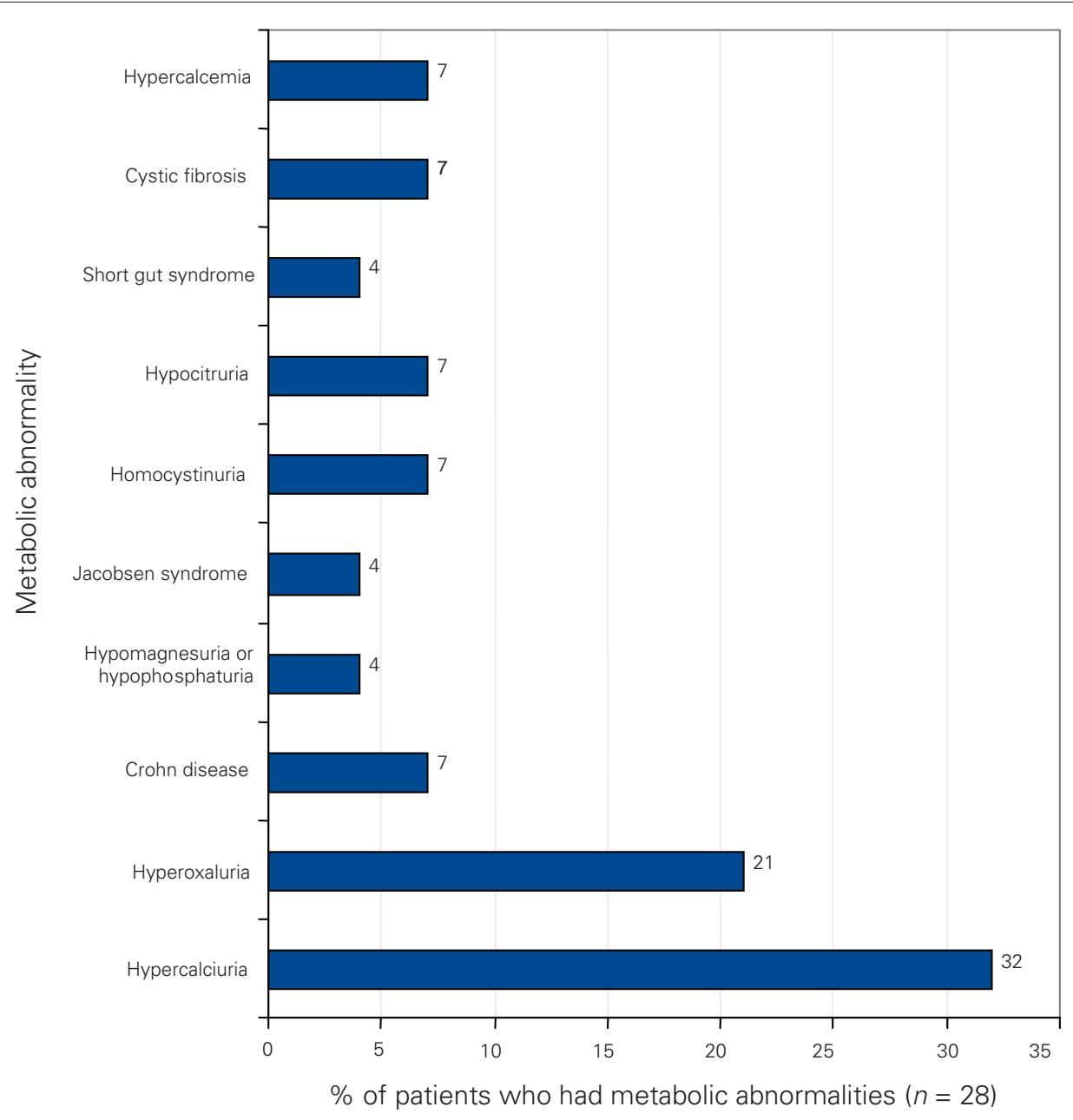

Fig. 3. Metabolic abnormalities of pediatric patients who underwent metabolic investigation for newly identified urolithiasis. 
ultrasonography and intravenous urography had a much higher degree of sensitivity for urolithiasis compared with kidney, ureter, and bladder radiography. Plain film radiography, however, can highlight other causes of abdominal pain or gastrointestinal complaints, such as constipation. Ultrasonography advantageously avoids radiation or intravenous contrast exposure. Urinary obstruction and extragenitourinary lesions can also be detected with ultrasonography. Ultrasonography is therefore a reasonable first choice for imaging a pediatric patient with suspected stone disease. If there is no stone seen on ultrasonography and a high degree of clinical suspicion remains, a CT scan should be obtained.

Bilateral stone disease was uncommon in our study, but occurred more often in the setting of an underlying metabolic abnormality. Rates of metabolic abnormalities in pediatric stone formers have been quoted as $48 \%-68 \%, 5,16,17$ and we observed a metabolic abnormality rate of $40 \%$. The most common abnormality identified was hypercalciuria, accounting for $32 \%$ of patients with metabolic problems. Pietrow and colleagues ${ }^{16}$ and Perrone and coworkers ${ }^{17}$ identified idiopathic hypercalciuria as the most common abnormality in children with urinary stones in developed countries. In contrast, hypocitruria and hypomagnesuria are the predominant abnormalities in Turkey and Pakistan. ${ }^{1,4,18}$ Although the etiology of the hypercalciuria found in our patients was not specifically assessed, there were no cases of renal tubular acidosis or hyperparathyroidism in any of our hypercalciuric patients. The observed high rates of metabolic abnormalities necessitate a full metabolic work up of these patients.

Stones recurred in $24 \%$ of patients during the mean 2.8-year follow-up, similar to the $19 \%$ recurrence rate found in the Pietrow series. ${ }^{16}$ Rates of $20 \%$ to $48 \%$ have also been reported, $, 5,16,17$ and our rate may have been higher given a longer period of follow-up. Of recurrences, $71 \%$ occurred in patients with a metabolic abnormality, which falls into the $30 \%$ to $95 \%$ range quoted in the literature. ${ }^{16,17}$ This underscores the importance of metabolic evaluation in first-time pediatric stone formers for early, appropriate treatment and close follow-up for recurrence.

Of our patients with stones, $14 \%$ had an anatomic genitourinary abnormality concurrent with stone formation. Ureteropelvic junction obstruction was the most common abnormality detected. Interestingly, there was no recurrent stone formation in this group.

Mean stone size in our series was $5 \mathrm{~mm}$, and in adults such stones have up to a $98 \%$ chance of spontaneous passage. ${ }^{16}$ Van Savage and colleagues ${ }^{13}$ showed a decrease in the stone passage rate in children with stones larger than $3 \mathrm{~mm}$, whereas Pietrow and colleagues ${ }^{16}$ showed a decrease occurring with stones larger than $4 \mathrm{~mm}$. In our study, the mean size of spontaneously passed stones was $4 \mathrm{~mm}$; stones requiring intervention were about twice as large, at $9 \mathrm{~mm}$. Our overall rate of spontaneous stone passage was $47 \%$, which is in keeping with the general observation of $50 \%$ in children with urinary calculi. ${ }^{3,9}$

The size, location and composition of stone, and urinary tract anatomy all factor into the choice of surgical therapy for persistent urinary calculi. ESWL has become the mainstay of treatment of symptomatic upper urinary tract calculi in children $n^{3,7}$ and was the first line of surgical intervention in $56 \%$ of our patients who did not pass calculi on their own. ESWL is effective in eliminating calculi in children without complications and with minimal radiation

\begin{tabular}{lc}
\hline $\begin{array}{l}\text { Table 1. Genitourinary anatomic abnormalities present in } \\
\text { 10 patients }\end{array}$ & $\begin{array}{c}\text { No. of } \\
\text { patients }\end{array}$ \\
\hline & 1 \\
Anatomic abnormality & 1 \\
\hline Chronic hydronephrosis with ureterocoele & 1 \\
Bifid left pelvis, tortuous ureter & 1 \\
Duplication of collecting system & 1 \\
Cloacal exstrophy & 1 \\
Bilateral hypodysplastic kidneys (Jacobsen syndrome) & 4 \\
Vesicoureteric reflux & Ureteropelvic junction obstruction
\end{tabular}

Table 2. Stone recurrence in 17 patients

\begin{tabular}{lc}
\hline Etiology of stone formation & $\begin{array}{c}\text { No. of } \\
\text { patients }\end{array}$ \\
\hline Hyperoxaluria & 4 \\
Hypercalciuria & 3 \\
Homozygous cystinuria & 2 \\
Short gut syndrome & 1 \\
Hypercalcemia & 1 \\
Crohn disease & 1 \\
None known & 5 \\
\hline
\end{tabular}


exposure. ${ }^{3}$ ESWL was unsuccessful in 3 of our patients, and 2 required percutaneous nephrolithotomies and the other ureteroscopy for stone clearance. Six (24\%) patients underwent ureteroscopy, with good result. Success rates for ureteroscopic laser lithotripsy and stone extraction for children with distal ureteral calculi vary from $86 \%$ to $100 \% .^{13}$ Small working channels, diminutive equipment and fragile tissues make this a challenging procedure. These children are at risk for ureteral stricture, postdilation vesicoureteric reflux and urethral stricture, complications whose risks are currently unknown. Seven (28\%) patients required percutaneous nephrolithotomies to eliminate their stones. Although a less invasive technique than open surgical management, long-term studies have yet to determine the effects of percutaneous nephrolithotomy on the developing kidney. ${ }^{19}$

The 42 stones collected and analyzed showed that $93 \%$ were calcium oxalate or phosphate stones, with $5 \%$ being cystine and $2 \%$ uric acid. Some publications describe the prevalence of cystine stones as $2 \%-3 \%$ and uric acid stones as $8 \%$ in children. ${ }^{7}$ Infection stones account for $2 \%-3 \%$ of the stones in pediatric patients. Although 10 (14\%) of our patients had bacteriuria, there were no actual infection stones detected. Similarly, previous studies by Marquardt and Nagel, ${ }^{20}$ and Milliner and Murphy ${ }^{5}$ have shown urinary tract infections associated with $14 \%-20 \%$ of pediatric stones.

\section{Conclusion}

Pediatric patients with stones present in a manner similar to adults. Primary care physicians need to be highly suspicious of stone disease in a patient presenting with flank or abdominal pain and gastrointestinal symptoms. Abdominal ultrasonography and plain film radiography are the preferred initial radiological investigations in children as they limit radiation exposure. CT scan may be performed secondarily if more sensitivity is required. Metabolic abnormalities are common in pediatric stone patients and are strongly related to recurrence. Anatomic abnormalities may coexist. Children must be investigated for these at their first diagnosis of stone disease. One-half of all patients will pass their stones spontaneously, especially if the stone size is less than $5 \mathrm{~mm}$. Available surgical options are similar to those for adults. Over $90 \%$ of pediatric stones are composed of calcium oxalate or phosphate, and recurrence rates are high. Therefore, long-term follow-up and patient and parent education regarding the importance of increased fluid intake and compliance with medical treatments are necessary.

From the Divisions of *Pediatric Urology and †Pediatric Nephrology, Children's Hospital of Eastern Ontario, University of Ottawa, Ottawa, Ont.

This article has been peer reviewed.

Competing interests: None declared.

\section{References}

1. Rizvi SAH, Naqui SA, Hussain Z, et al. Pediatric urolithiasis: developing nations perspectives. J Urol 2002;168:1522-5.

2. Marx JA, Hockberger S, Walls RM, et al, editors. Rosen's emergency medicine: concepts and clinical practice. 5th ed. St. Louis (MO): Mosby; 2002. p. 2234.

3. Kroovand RL. Pediatric urolithiasis. Urol Clin North Am 1997;24:173-84.

4. Erbagci A, Erbagci, AB, Yilmaz M, et al. Pediatric urolithiasis. Scand J Urol Nephrol 2002; 37:129-33.

5. Milliner DS, Murphy ME. Urolithiasis in pediatic patients. Mayo Clin Proc 1993;68:241-8.

6. Sarkissan A, Babloyan A, Arikyants N, et al. Pediatric urolithiasis in Armenia: a study of 198 patients observed from 1991 to 1999. Pediatr Nephrol 2001;16:728-32.

7. Minevich E. Pediatric urolithiasis. Pediatr Clin North Am 2001;48:1571-86.

8. Grossfield PD, Mattina T, Lai Z, et al. The $11 \mathrm{q}$ terminal deletion disorder: a prospective study of 110 cases. Am J Med Genet 2004;129:51-61.

9. Gearhart J, Herzberg G, Jeffs R. Childhood urolithiasis: experience and advances. Pediatrics 1991;87:445-9.

10. Ece A, Ozdemir E, Gurkan $F$, et al. Characteristics of pediatric urolithiasis in southeast Anatolia. Int J Urol 2000;7:330-4.

11. Ozokutan BH, Kucukaydin M, Gunduz Z, et al. Urolithiasis in childhood. Pediatr Surg Int 2000; 16:60-3.

12. Battino B, DeFoor $\mathrm{W}$, Coe F, et al. Metabolic evaluation of children with urolithiasis: Are adult references for supersaturation appropriate? J Urol 2002;168:2568-71.

13. Van Savage JG, Palanaca LG, Andersen RD, et al. Treatment of distal ureteral stones in children: similarities to the AUA guidelines in adults. J Urol 2000;164:1089-93.

14. Walther PC, Lamm D, Kaplan GW, et al. Pediatric urolithiasis: a ten year review. Pediatrics 1980;65:1068-72

15. Wiest PW, Locken JA, Heintz PH, et al. CT scanning: a major source of radiation exposure. Semin Ultrasound CT MR 2002;5:402-10.

16. Pietrow P, Pope JC 4th, Adams MC, et al. Clinical outcome of pediatric stone disease. J Urol 2002;167:670-3.

17. Perrone HC, dos Santos DR, Santos MV, et al. Urolithiasis in childhood: metabolic evaluation. Pediatr Nephrol 1992;6:54-6.

18. Tekin A, Tekqul S, Atsu N. et al. A study of the etiology of idiopathic calcium urolithiasis in children: hypocitruria is the most important risk factor. J Urol 2002;164:162-5.

19. Jayanthi VR, Arnold PM, Koff SA. Strategies for managing upper tract calculi in young children. J Urol 1999;162:1234-7.

20. Marquardt H, Nagel R. Urolithiasis in childhood. Urology 1977;9:627-9.

Correspondence: Dr. Michael Leonard, Division of Urology, Children's Hospital of Eastern Ontario, 401 Smyth Rd., Ottawa ON K1H 8L1; mleonard@cheo.on.ca 